\title{
Cryptanalysis of Full RIPEMD-128
}

\author{
Franck Landelle ${ }^{1}$ and Thomas Peyrin ${ }^{2, \star}$ \\ 1 DGA MI, France \\ 2 Division of Mathematical Sciences, School of Physical and Mathematical Sciences, \\ Nanyang Technological University, Singapore \\ landelle.franck@laposte.net, thomas.peyrin@gmail.com
}

\begin{abstract}
In this article we propose a new cryptanalysis method for double-branch hash functions that we apply on the standard RIPEMD-128, greatly improving over know results. Namely, we were able to build a very good differential path by placing one non-linear differential part in each computation branch of the RIPEMD-128 compression function, but not necessarily in the early steps. In order to handle the low differential probability induced by the non-linear part located in later steps, we propose a new method for using the freedom degrees, by attacking each branch separately and then merging them with free message blocks. Overall, we present the first collision attack on the full RIPEMD-128 compression function as well as the first distinguisher on the full RIPEMD-128 hash function. Experiments on reduced number of rounds were conducted, confirming our reasoning and complexity analysis. Our results show that 16 years old RIPEMD-128, one of the last unbroken primitives belonging to the MD-SHA family, might not be as secure as originally thought.
\end{abstract}

Keywords: RIPEMD-128, collision, distinguisher, hash function.

\section{Introduction}

Recent impressive progresses in hash function cryptanalysis [25 28 29 27] led to the fall of most standardized primitives, such as MD4, MD5, SHA-0 and SHA-1. All these algorithms share the same design rationale for their compression functions (i.e. they incorporate additions, rotations, xors and boolean functions in an unbalanced Feistel network), and we usually refer to them as the MD-SHA family. As of today, among this family only SHA-2, RIPEMD-128 and RIPEMD-160 remain unbroken.

The notation RIPEMD represents several distinct hash functions related to the MD-SHA-family, the first representative being RIPEMD-0 [2] that was recommended in 1992 by the European RACE Integrity Primitives Evaluation (RIPE) consortium. Its compression function basically consists in two MD4-like 20] functions computed in parallel (but with different constant additions for the two branches), with 48 steps in total. Early cryptanalysis by Dobbertin on a reduced version

\footnotetext{
* Supported by the Singapore National Research Foundation Fellowship 2012 (NRFNRFF2012-06).
} 
of the compression function 9] seemed to indicate that RIPEMD-0 was a weak function and this was fully confirmed much later by Wang et al. 27. who showed that one can find a collision for the full RIPEMD-0 hash function with as few as $2^{16}$ computations.

However, in 1996, due to the cryptanalysis advances on MD4 and on the compression function of RIPEMD-0, the original RIPEMD-0 was reinforced by Dobbertin, Bosselaers and Preneel [10] to create two stronger primitives RIPEMD-128 and RIPEMD-160, with 128/160-bit output and 64/80 steps respectively (two other less known 256 and 320-bit output variants RIPEMD-256 and RIPEMD-320 were also proposed, but with a claimed security level equivalent to an ideal hash function with a twice smaller output size). The main novelty compared to RIPEMD-0 is that the two computation branches were made much more distinct by using not only different constants, but also different rotation values and boolean functions, which greatly hardens the attacker's task in finding good differential paths for both branches at a time. The security seems to have indeed increased since as of today no attack is known on the full RIPEMD-128 or RIPEMD-160 compression/hash functions and the two primitives are worldwide ISO/IEC standards [12].

Even though no result is known on the full RIPEMD-128 and RIPEMD-160 compression/hash functions yet, many analysis were conducted in the recent years. In [17, a preliminary study checked up to what extent can the known attacks [27] on RIPEMD-0 apply to RIPEMD-128 and RIPEMD-160. Then, following the extensive work on preimage attacks for MD-SHA family, 211926] describe high complexity preimage attacks on up to 36 steps of RIPEMD-128 and 31 steps of RIPEMD-160. Collision attacks were considered in [16] for RIPEMD-128 and in [15] for RIPEMD-160, with 48 and 36 steps broken respectively. Finally, distinguishers based on non-random properties such as second-order collisions are given in [16 22 15], reaching about 50 steps with a very high complexity.

Our Contributions. In this article, we introduce a new type of differential path for RIPEMD-128 using one non-linear differential trail for both left and right branches and, in contrary to previous work, not necessarily located in the early steps (Section 3). The important differential complexity cost of these two parts is mostly avoided by using the freedom degrees in a novel way: some message words are used to handle the non-linear parts in both branches and the remaining ones are used to merge the internal states of the two branches (Section 44). Overall, we obtain the first cryptanalysis of the full 64-round RIPEMD-128 hash and compression functions. Namely, we provide a distinguisher based on a differential property for both the full 64-round RIPEMD-128 compression function and hash function (Section 5). Previously best-known results for non-randomness properties only applied to 52 steps of the compression function, 48 steps of the hash function. More importantly, we also derive a semi-free-start (SFS) collision attack on the full RIPEMD-128 compression function (Section 5), significantly improving the previous free-start (FS) collision attack on 48 steps. Any further improvement of our techniques is likely to provide a practical SFS collision attack on the RIPEMD-128 compression function. In order to increase the confidence in 
Table 1. Summary of known and new results on RIPEMD-128 hash function

\begin{tabular}{|c|c|c|c|c|c|c|}
\hline Function & Size & Key Setting & Target & \#Steps & Complexity & Ref. \\
\hline RIPEMD-128 & 128 & comp. function & preimage & 35 & $2^{112}$ & 19 \\
\hline RIPEMD-128 & 128 & hash function & preimage & 35 & $2^{121}$ & 19 \\
\hline RIPEMD- 128 & 128 & hash function & preimage & 36 & & 26 \\
\hline$\overline{\mathrm{R}} \overline{\mathrm{IP}} \overline{\mathrm{EM}} \overline{\mathrm{D}}-\overline{1} \overline{28}$ & $\overline{1} 2 \overline{8}$ & comp. $\overline{\text { function }}$ & collision & $\overline{48}$ & $-\overline{2}^{4 \sigma}$ & 16 \\
\hline RIPEMD-128 & 128 & comp. function & collision & 60 & $2^{57.57}$ & new \\
\hline RIPEMD-128 & 128 & comp. function & collision & 63 & $2^{59.91}$ & new \\
\hline RIPEMD-128 & 128 & comp. function & collision & Full & $2^{61.57}$ & new \\
\hline RIPEMD- 128 & 128 & hash function & collision & 38 & $2^{14}$ & 16 \\
\hline $\bar{R} \overline{I P E M D}-\overline{1} \overline{28}$ & $\overline{1} 2 \overline{8}$ & $-\overline{c o m p}$. function & non-rand. & $5 \overline{2}$ & $2^{107}$ & 22 \\
\hline RIPEMD-128 & 128 & comp. function & non-rand. & Full & $2^{59.57}$ & new \\
\hline RIPEMD -128 & 128 & hash function & non-rand. & 48 & $2^{70}$ & 16 \\
\hline RIPEMD- 128 & 128 & hash. function & non-rand. & Full & $2^{105.40}$ & new \\
\hline
\end{tabular}

our reasoning, we implemented independently the two main parts of the attack (the merge and the probabilistic part) and the observed complexity matched our predictions. Our results and previous works complexities are given in Table 1 for comparison.

\section{Description of RIPEMD-128}

RIPEMD-128 [10] is a 128-bit hash function that uses the Merkle-Damgård construction as domain extension algorithm: the hash function $H$ is built by iterating a 128-bit compression function $h$ that takes as input a 512-bit message block $m_{i}$ and a 128-bit chaining variable $c v_{i}: c v_{i+1}=h\left(c v_{i}, m_{i}\right)$, where the message $m$ to hash is padded beforehand to a multiple of 512 bits 1 and the first chaining variable is set to a predetermined initial value $c v_{0}=I \mathrm{~V}$.

We refer to 10 for a complete description of RIPEMD-128. In the rest of this article, we denote by $[Z]_{i}$ the $i$-th bit of a word $Z$, starting the counting from 0 . $\boxplus$ and $\boxminus$ represent the modular addition and subtraction on 32 bits, and $\oplus, \vee$, $\wedge$, the bitwise "exclusive or", the bitwise "or", and the bitwise "and" function respectively.

\subsection{RIPEMD-128 Compression Function}

The RIPEMD-128 compression function is based on MD4, with the particularity that it uses two parallel instances of it. We differentiate these two computation branches by left and right branch and we denote by $X_{i}\left(\operatorname{resp} . Y_{i}\right.$ ) the 32-bit word of left branch (resp. right branch) that will be updated during step $i$ of the compression function. The process is composed of 64 steps divided into 4 rounds of 16 steps each in both branches.

Initialization. The 128-bit input chaining variable $c v_{i}$ is divided into 4 words $h_{i}$ of 32 bits each, that will be used to initialize the left and right branch 128-bit

\footnotetext{
${ }^{1}$ The padding is the same as for MD4: a "1" is first appended to the message, then $x$ "0" bits (with $x=512-(|m|+1+64(\bmod 512))$ ) are added, and finally the message length $|m|$ coded on 64 bits is appended as well.
} 
internal state: $X_{-3}=h_{0}, X_{-2}=h_{1}, X_{-1}=h_{2}, X_{0}=h_{3}, Y_{-3}=h_{0}, Y_{-2}=h_{1}$, $Y_{-1}=h_{2}, Y_{0}=h_{3}$

The Message Expansion. The 512-bit input message block is divided into 16 words $M_{i}$ of 32 bits each. Each word $M_{i}$ will be used once in every round in a permuted order (similarly to MD4) and for both branches. We denote by $W_{i}^{l}$ (resp. $W_{i}^{r}$ ) the 32-bit expanded message word that will be used to update the left branch (resp. right branch) during step $i$. We have for $0 \leq j \leq 3$ and $0 \leq k \leq 15: W_{j \cdot 16+k}^{l}=M_{\pi_{j}^{l}(k)}$ and $W_{j \cdot 16+k}^{r}=M_{\pi_{j}^{r}(k)}$, where $\pi_{j}^{l}$ and $\pi_{j}^{r}$ are permutations.

The Step Function. At every step $i$, the registers $X_{i+1}$ and $Y_{i+1}$ are updated with functions $f_{j}^{l}$ and $f_{j}^{r}$ that depends on the round $j$ in which $i$ belongs:

$$
\begin{aligned}
X_{i+1} & =\left(X_{i-3} \boxplus \Phi_{j}^{l}\left(X_{i}, X_{i-1}, X_{i-2}\right) \boxplus W_{i}^{l} \boxplus K_{j}^{l}\right) \lll s_{i}^{l}, \\
Y_{i+1} & =\left(Y_{i-3} \boxplus \Phi_{j}^{r}\left(Y_{i}, Y_{i-1}, Y_{i-2}\right) \boxplus W_{i}^{r} \boxplus K_{j}^{r}\right)^{\lll s_{i}^{r},}
\end{aligned}
$$

where $K_{j}^{l}, K_{j}^{r}$ are 32 -bit constants defined for every round $j$ and every branch, $s_{i}^{l}, s_{i}^{r}$ are rotation constants defined for every step $i$ and every branch, $\Phi_{j}^{l}, \Phi_{j}^{r}$ are 32 -bit boolean functions defined for every round $j$ and every branch. All these constants and functions, as well as the $I V$ and the permutations $\pi_{j}^{l}$ and $\pi_{j}^{r}$ can be found in the original RIPEMD-128 documentation [10.

The Finalization. A finalization and a feed-forward is applied when all 64 steps have been computed in both branches. The four 32-bit words $h_{i}^{\prime}$ composing the output chaining variable are finally obtained by: $h_{0}^{\prime}=X_{63} \boxplus Y_{62} \boxplus h_{1}$, $h_{1}^{\prime}=X_{62} \boxplus Y_{61} \boxplus h_{2}, h_{2}^{\prime}=X_{61} \boxplus Y_{64} \boxplus h_{3}, h_{3}^{\prime}=X_{64} \boxplus Y_{63} \boxplus h_{0}$

\section{A New Family of Differential Paths for RIPEMD-128}

\subsection{The General Strategy}

The first task for an attacker looking for collisions in some compression function is to set a good differential path. In the case of RIPEMD and more generally double or multi-branches compression functions, this can be quite a difficult task because the attacker has to find a good path for all branches at the same time. This is exactly what multi-branches functions designers are hoping: it is unlikely that good differential paths exist in both branches at the same time when the branches are made distinct enough (note that the weakness of RIPEMD-0 is that both branches are almost identical and the same differential path can be used for the two branches at the same time).

Differential paths in recent collision attacks on MD-SHA family are composed of two parts: a low probability non-linear part in the first steps and a high probability linear part in the remaining ones. Only the latter will be handled probabilistically and impact the overall complexity of the collision finding algorithm, since during the first steps the attacker can choose message words independently. 
This strategy proved to be very effective because it allows to find much better linear parts than before by relaxing many constraints on them. The previous approaches for attacking RIPEMD-128 [17/16] are based on the same strategy, building good linear paths for both branches, but without including the first round (i.e. the first 16 steps). The first round in each branch will be covered by a non-linear differential path and this is depicted left in Figure 1. The collision search is then composed of two subparts, the first handling the low-probability non-linear paths with the message blocks (step (1) and then the remaining steps in both branches are verified probabilistically (step (2)).
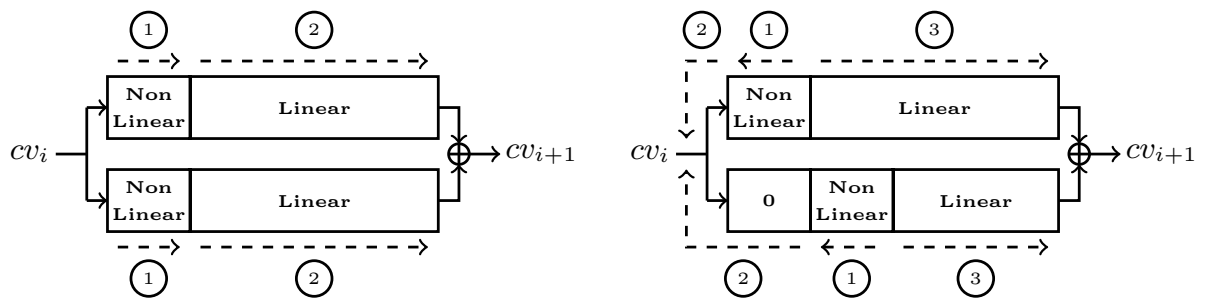

Fig. 1. The previous (left-hand side) and new (right-hand side) approach for collision search on double-branch compression functions

This differential path search strategy is natural when one will handle the non-linear parts in a classic way (i.e. computing only forward) during the collision search, but in Section 4 we will describe a new approach for using the available freedom degrees provided by the message words in double-branch compression functions (see right in Figure 1): instead of handling the first rounds of both branches at the same time during the collision search, we will satisfy them independently (step (1)), then use some remaining free message words to merge the two branches (step (2) and finally handle the remaining steps in both branches probabilistically (step (3). This new approach broadens the search area of good linear differential parts, and provides us better candidates in the case of RIPEMD-128.

\subsection{Finding a Good Linear Part}

Since any active bit in a linear differential path (i.e. a bit containing a difference) is likely to cause many conditions in order to control its spread, most successful collision searches start with a low-weight linear differential path, therefore reducing the complexity as much as possible. RIPEMD-128 is no exception, and because every message word is used once in every round of every branch in RIPEMD-128, the best would be to insert only a single-bit difference in one of them. This was considered in [16], but the authors concluded that none of all single-word differences leads to a good choice and they eventually had to utilize one active bit in two message words instead, therefore doubling the amount of differences inserted during the compression function computation and reducing the overall number of steps they could attack. By relaxing the constraint that 
both non-linear parts must necessarily be located in the first round, we show that a single-word difference in $M_{14}$ is actually a very good choice.

Boolean Functions. Analyzing the various boolean functions in RIPEMD-128 rounds is very important. Indeed, there are three distinct functions: XOR, ONX and IF, with all very distinct behavior. The function IF is non-linear and can absorb differences (one difference on one of its input can be blocked from spreading to the output by setting some appropriate bit value conditions). In other words, one bit difference in the internal state during an IF round can be forced to create only a single bit difference 4 steps later, thus providing no diffusion at all. In the contrary, XOR is arguably to most problematic function in our situation because it can not absorb any difference. Thus, one bit difference in the internal state during an XOR round will double the number of bit differences every step and quickly lead to an unmanageable amount of conditions. Moreover, the linearity of the XOR function makes it problematic when using the non-linear part search tool that strongly leverages non-linear behavior to obtain a solution. In between, the ONX function is non-linear for two inputs and can absorb difference up to some extent. We can easily conclude that the goal for the attacker will be to locate the biggest proportion of differences in the IF or if needed in the ONX functions, and try to avoid the XOR parts as much as possible.

Choosing a Message Word. We would like to find the best choice for the single-message word difference insertion. The XOR function located in the $4^{\text {th }}$ round of right branch must be avoided, so we are looking for a message word that is incorporated either very early (so we can propagate the difference backward) or very late (so we can propagate the difference forward) in this round. Similarly, the XOR function located in the $1^{\text {st }}$ round of left branch must be avoided, so we are looking for a message word that is incorporated either very early (for a FS collision attack) or very late (for a SFS collision attack) in this round as well. It is easy to check that $M_{14}$ is a perfect candidate, being the last inserted in $4^{\text {th }}$ round of right branch and the second-to-last in $1^{\text {st }}$ round of left branch.

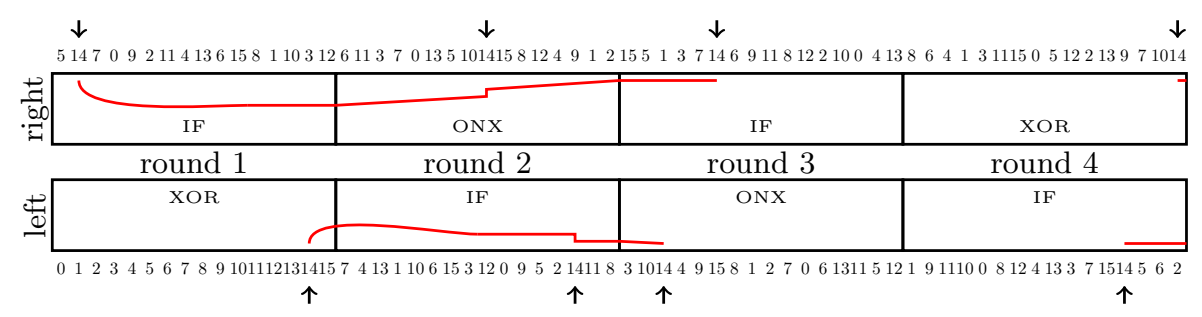

Fig. 2. The shape of our differential path for RIPEMD-128. The numbers are the message words inserted at each step and the red curves represent the rough amount differences in the internal state during each steps. The arrows show where the bit differences are injected with $M_{14}$.

Building the Linear Part. Once we chose that the only message difference will be a single bit in $M_{14}$, we need to build the whole linear part of the differential path in the internal state. By linear we mean that all modular additions will 
be modeled as a bitwise XOR function. Moreover, when a difference is input of a boolean function, it is absorbed when possible in order to remain as low weight as possible (though, for a few special bit positions it might be more interesting to not absorb the difference if it can erase another difference in later steps). We give the rough skeleton of our differential path in Figure 2, Both differences inserted in the $4^{\text {th }}$ round of left and right branches are simply propagated forward for a few steps and we are very lucky that this linear propagation leads to two final internal states whose difference can be mutually erased after application of the compression function finalization and feed-forward. All differences inserted in the $3^{r d}$ and $2^{\text {nd }}$ rounds of left and right branches are propagated linearly backward and will be later connected to the bit difference inserted in the $1^{\text {st }}$ round by the non-linear part. Note that since a non-linear part usually has a low differential probability, we will try to make it as thin as possible. No difference will be present in the input chaining variable, so the trail is well suited for a SFS collision attack. We had to choose the bit position for the message $M_{14}$ difference insertion and among the 32 possible choices, the most significant bit was selected because it is the one maximizing the differential probability of the linear part we just built (this finds an explanation by the fact that at the most significant bit position many conditions due to carry control in modular additions are avoided).

\subsection{The Non-linear Differential Part Search Tool}

Finding non-linear differential path is a very complex task, but we implemented a tool similar to [4 for SHA-1 in order to perform this task in an automated way. Since RIPEMD-128 also belongs to the MD-SHA family, the original technique works well, in particular when used in a round with a non-linear boolean function such as IF.

We have to find one non-linear part in each branch and note that they can be handled independently. We included the special constraint that the non-linear parts should be as thin as possible (i.e. spreading on the fewer possible amount of steps), so as to later reduce the overall complexity (linear parts have higher differential probability than non-linear ones).

\subsection{The Final Differential Path Skeleton}

Applying our non-linear part search tool and reusing notations from [4, we obtain the differential path in Figure 3, for which we provide at each step $i$ the differential probability $\mathrm{P}^{l}[i]$ and $\mathrm{P}^{r}[i]$ of left and right branch respectively. Also, we give for each step $i$ the accumulated probability $\mathrm{P}[i]$ starting from last step, i.e. $\mathrm{P}[i]=\prod_{j=63}^{j=i}\left(\mathrm{P}^{r}[j] \cdot \mathrm{P}^{l}[j]\right)$.

One can check that the trail has differential probability $2^{-85.09}$ (that is $\left.\prod_{i=0}^{63} \mathrm{P}^{l}[i]=2^{-85.09}\right)$ in the left branch and $2^{-145}\left(\right.$ i.e. $\prod_{i=0}^{63} \mathrm{P}^{r}[i]=2^{-145}$ ) in the right branch. Its overall differential probability is $2^{-230.09}$ and since we have 511 bits of message with unspecified value (one bit of $M_{4}$ is already set to " 1 "), plus 127 unrestricted bits of chaining variable (one bit of $X_{0}=Y_{0}=h_{3}$ is already set to "0"), we expect many solutions to exist (about $2^{407.91}$ ). 


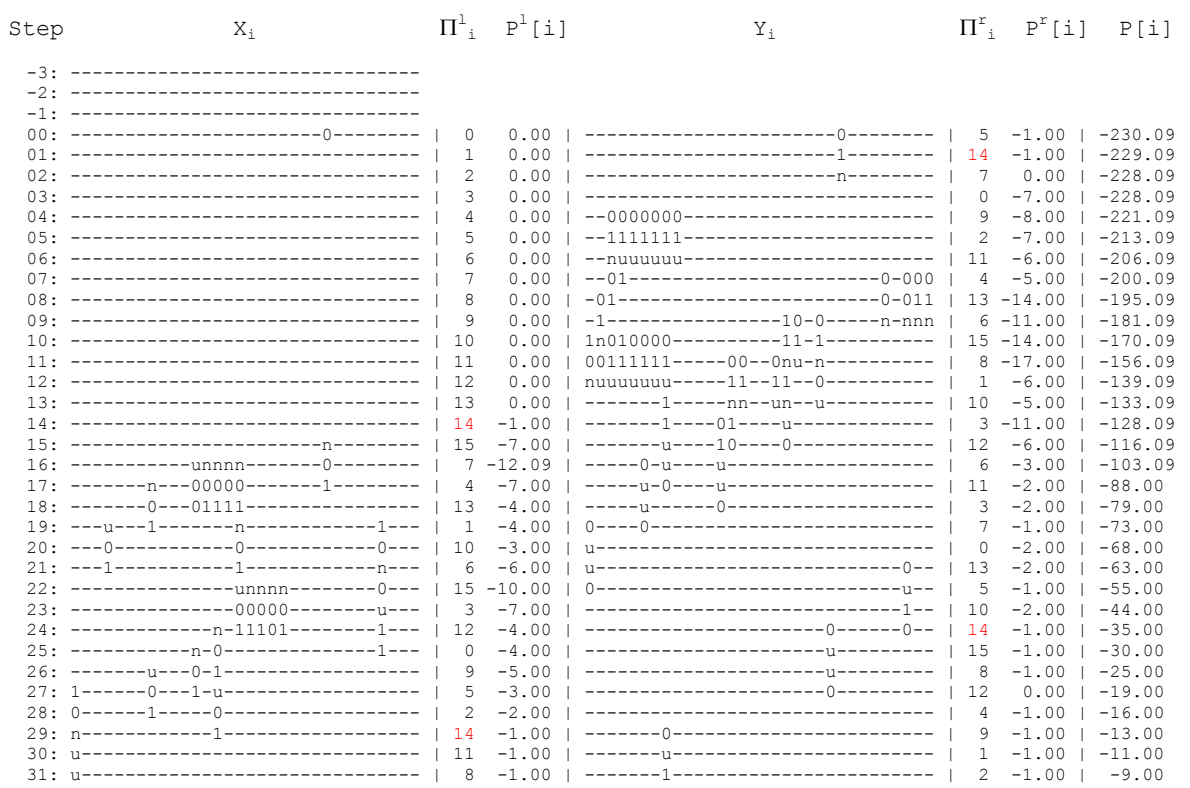

Fig. 3. The differential path for RIPEMD-128, after the non-linear parts search. The notations are the same as in $\left[4\right.$ and $\mathrm{P}^{l}[i], \mathrm{P}^{r}[i]$ and $P[i]$ are given in $\log _{2}()$.

In order for the path to provide a collision, the bit difference in $X_{61}$ must erase the one in $Y_{64}$ during the finalization phase of the compression function: $h_{2}^{\prime}=$ $X_{61} \boxplus Y_{64} \boxplus h_{3}$. Since the signs of these two bit differences are not specified, this happens with probability $2^{-1}$ and the overall probability to follow our differential path and to obtain a collision for a randomly chosen input is $2^{-231.09}$.

\section{Utilization of the Freedom Degrees}

In the differential path from Figure 3, the difference mask is already entirely set, but almost all message bits and chaining variable bits have no constraint with regards to their value. All these freedom degrees can be used to reduce the complexity of the straightforward collision search (i.e. choosing random 512-bit message values) that requires about $2^{231.09}$ RIPEMD-128 step computations. We will utilize these freedom degrees in three phases:

- Phase 1: we first fix some internal state and message bits in order to prepare the attack. This will allow us to handle in advance some conditions in the differential path as well as facilitating the merging phase. This preparation phase is done once for all.

- Phase 2: we will fix iteratively the internal state words $X_{21}, X_{22}, X_{23}, X_{24}$ from left branch, and $Y_{11}, Y_{12}, Y_{13}, Y_{14}$ from right branch, as well as message words $M_{12}, M_{3}, M_{10}, M_{1}, M_{8}, M_{15}, M_{6}, M_{13}, M_{4}, M_{11}$ and $M_{7}$ (the ordering 
is important). This will provide us a starting point for the merging phase and due to a lack of freedom degrees, we will need to perform this phase several times in order to get enough starting points to eventually find a solution for the entire differential path.

- Phase 3: we use the remaining unrestricted message words $M_{0}, M_{2}, M_{5}$, $M_{9}$ and $M_{14}$ to efficiently merge the internal states of the left and right branches.

\subsection{Phase 1: Preparation}

Before starting to fix a lot of message and internal state bit values, we need to prepare the differential path from Figure 3 so that the merge can later be done efficiently and so that the probabilistic part will not be too costly. Understanding these constraints requires a deep insight of the differences propagation and conditions fulfillment inside the RIPEMD-128 step function. Therefore, the reader not interested in the details of the differential path construction is advised to skip this subsection.

The first constraint that we set is $Y_{3}=Y_{4}$. The effect is that the IF function at step 4 of the right branch, $\operatorname{IF}\left(Y_{2}, Y_{4}, Y_{3}\right)=\left(Y_{2} \wedge Y_{3}\right) \oplus\left(\overline{Y_{2}} \wedge Y_{4}\right)=Y_{3}=Y_{4}$, will not depend on $Y_{2}$ anymore. We will see in Section 4.3 that this constraint is crucial in order for the merge to be performed efficiently.

The second constraint is $X_{24}=X_{25}$ (except the two bit positions of $X_{24}$ and $X_{25}$ that contain differences), and the effect is that the IF function at step 26 of the left branch (when computing $\left.X_{27}\right), \operatorname{IF}\left(X_{26}, X_{25}, X_{24}\right)=\left(X_{26} \wedge X_{25}\right) \oplus\left(\overline{X_{26}} \wedge\right.$ $\left.X_{24}\right)=X_{24}=X_{25}$, will not depend on $X_{26}$ anymore. Before the final merging phase starts, we will not know $M_{0}$, and having this $X_{24}=X_{25}$ constraint will allow us to directly fix the conditions located on $X_{27}$ without knowing $M_{0}$ (since $X_{26}$ directly depends on $M_{0}$ ). Moreover, we fix the 12 first bits of $X_{23}$ and $X_{24}$ to "01000100u001" and "01000011110" respectively because this choice is among the few that minimizes the number of bits of $M_{9}$ that needs to be set in order to verify many of the conditions located on $X_{27}$.

The third constraint consists in setting the bits 18 to 30 of $Y_{20}$ to zero. The effect is that for these 13 bit positions, the ONX function at step 21 of the right branch (when computing $\left.Y_{22}\right), \operatorname{ONX}\left(Y_{21}, Y_{20}, Y_{19}\right)=\left(Y_{21} \vee \overline{Y_{20}}\right) \oplus Y_{19}$, will not depend on the 13 corresponding bits of $Y_{21}$ anymore. Again, because we will not know $M_{0}$ before the merging phase starts, this constraint will allow us to directly fix the conditions on $Y_{22}$ without knowing $M_{0}$ (since $Y_{21}$ directly depends on $\left.M_{0}\right)$.

Finally, the last constraint that we enforce is that the first two bits of $Y_{22}$ are set to " 10 " and the first three bits of $M_{14}$ are set to "011". This particular choice of bit values is among the ones that reduces the most the spectrum of possible carries during the addition of step 24 (when computing $Y_{25}$ ) and we obtain a probability improvement to reach " $u$ " in $Y_{25}$ from $2^{-1}$ to $2^{-0.25}$.

We observe that all the constraints set in this subsection consume in total $32+51+13+5=101$ bits of freedom degrees, and a huge amount of solutions (about $2^{306.91}$ ) are still expected to exist. 


\subsection{Phase 2: Generating a Starting Point}

Once the differential path properly prepared in phase 1, we would like to utilize the huge amount of freedom degrees available to fulfill directly as many conditions as possible. Our approach is to fix the value of the internal states in both the left and right branches (they can be handled independently), exactly in the middle of the non-linear parts where the number of conditions is important. Then, we will fix the message words one by one following a particular scheduling, and propagating the bit values forward and backward from the middle of the non-linear parts in both branches.

Fixing the Internal State. We chose to start by setting the values of $X_{21}$, $X_{22}, X_{23}, X_{24}$ in the left branch, and $Y_{11}, Y_{12}, Y_{13}, Y_{14}$ in the right branch, because they are located right in the middle of the non-linear parts. We take the first word $X_{21}$ and randomly set all of its unrestricted "_" bits to "0" or " 1 " and check if any direct inconsistency is created with this choice. If that is the case, we simply pick another candidate until no direct inconsistency is deduced. Otherwise, we can go to the next word $X_{22}$, etc. If too many tries are failing for a particular internal state word, we can backtrack and pick another choice for the previous word. Finally, if no solution is found after a certain amount of time, we just restart the whole process, so as to avoid being blocked in a particularly bad subspace with no solution.

Fixing the Message Words. Similarly to the internal state words, we randomly fix the value of message words $M_{12}, M_{3}, M_{10}, M_{1}, M_{8}, M_{15}, M_{6}, M_{13}$, $M_{4}, M_{11}$ and $M_{7}$ (following this particular ordering that facilitates the convergence towards a solution). The difference here is that the left and right branch computations are no more independent since the message words are used in both of them. However, this does not change anything to our algorithm and the very same process is applied: for each new message word randomly fixed, we compute forward and backward from the known internal state values and check for any inconsistency, using backtracking and reset if needed.

Overall, finding one new solution for this entire phase 2 takes about 5 minutes of computation on a recent $\mathrm{PC}$ with a naive implementation 2 . However, when one starting point is found, we can generate many for a very cheap cost by randomizing message words $M_{4}, M_{11}$ and $M_{7}$ since the most difficult part is to fix the 8 first message words of the schedule. For example, once a solution is found, one can directly generate $2^{18}$ new starting points by randomizing a certain portion of $M_{7}$ (because $M_{7}$ has no impact on the validity of the nonlinear part in the left branch, while in the right branch one has only to ensure that the last 14 bits of $Y_{20}$ are set to "u0000000000000") and this was verified experimentally.

${ }^{2}$ Our message word fixing approach is certainly not optimal, but this phase is not the bottleneck of our attack and we preferred to aim for simplicity when possible. In case a very fast implementation is needed, a more efficient but more complex strategy would be to find a bit per bit scheduling instead of a word-wise one. 
We give an example of such a starting point in Figure 4 and we emphasize that by "solution" or "starting point" we mean a differential path instance with exactly the same probability profile as this one. The 3 constrained bit values in $M_{14}$ are coming from the preparation in phase 1 , and the 3 constrained bit values in $M_{9}$ are necessary conditions in order to fulfill step 26 when computing $X_{27}$. It is also important to remark that whatever instance found during this second phase, the position of these 3 constrained bit values will always be the same thanks to our preparation in phase 1 .

The probabilities displayed in Figure 4 for early steps (steps 0 to 14) are not meaningful here since they assume an attacker only computing forward, while in our case we will compute backwards from the non-linear parts to the early steps. However, we can see that the uncontrolled accumulated probability (i.e. step (3) in right side of Figure (1) is now improved to $2^{-29.32}$, or $2^{-30.32}$ if we add the extra condition for the collision to happen at the end of the RIPEMD-128 compression function.
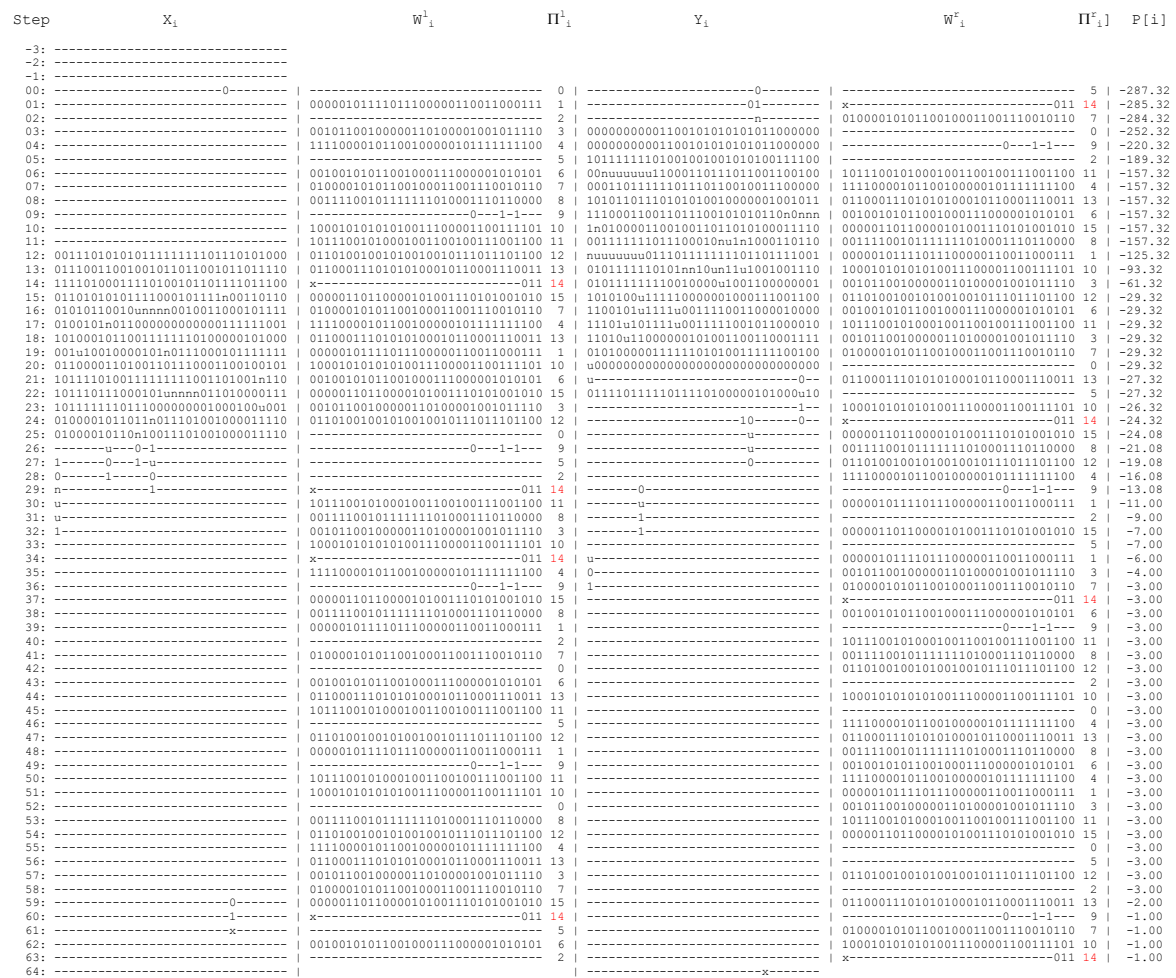

Fig. 4. The differential path for RIPEMD-128, after the second phase of the freedom degree utilization. The notations are the same as in [4] and $P[i]$ is given in $\log _{2}()$. 


\subsection{Phase 3: Merging Left and Right Branches}

At the end of the second phase, we have several starting points equivalent to the one from Figure 4, with many conditions already verified and an uncontrolled accumulated probability of $2^{-30.32}$. Our goal for this third phase is now to use remaining free message words $M_{0}, M_{2}, M_{5}, M_{9}, M_{14}$ and make sure that both left and right branches start with the same chaining variable.

We recall that during the first phase we enforced that $Y_{3}=Y_{4}$, and for the merge we will require an extra constraint $X_{5}^{\ggg 5} \boxminus M_{4}=0 x$ xfffffff. The message words $M_{14}$ and $M_{9}$ will be utilized to fulfill this constraint, and message words $M_{0}, M_{2}$ and $M_{5}$ will be used to perform the merge of the two branches only with a few operations, and with a success probability of $2^{-34}$.

Handling the Extra Constraint with $M_{14}$ and $M_{9}$. First, let us deal with the constraint $X_{5}^{\gg 5} \boxminus M_{4}=0$ xffffffff, which can be rewritten as $X_{5}=$ $\left(0 x\right.$ xfffffff $\left.\boxplus M_{4}\right) \lll 5$ and then $\left(0 x f f f f f f f \boxplus M_{4}\right)^{\lll 5}=X_{9}^{\ggg 11} \boxminus\left(X_{8} \oplus X_{7} \oplus\right.$ $\left.X_{6}\right) \boxminus M_{8} \boxminus K_{0}^{l}$ by replacing $M_{5}$ using update formula of step 8 in left branch. Finally, isolating and replacing $X_{6}$ using update formula of step 9 in left branch:

$$
M_{9}=X_{10}^{\ggg 13} \boxminus\left(\left(X_{9}^{\ggg 11} \boxminus M_{8} \boxminus K_{0}^{l} \boxminus\left(\text { oxffffffff } \boxplus M_{4}\right)^{\lll 5}\right) \oplus X_{8} \oplus X_{7}\right) \boxminus K_{0}^{l} \boxminus\left(X_{9} \oplus X_{8} \oplus X_{7}\right) .(1)
$$

All values on the right side of this equation are known if $M_{14}$ is fixed. Therefore, so as to fulfill our extra constraint, what we could do is to simply pick a random value for $M_{14}$, and then directly deduce the value of $M_{9}$ thanks to equation (11). However, one can see in Figure 4 that 3 bits are already fixed in $M_{9}$ (the last one being the $10^{\text {th }}$ bit of $M_{9}$ ) and thus a valid solution would be found only with probability $2^{-3}$. In order to avoid this extra complexity factor, we will first randomly fix the first 24 bits of $M_{14}$ and this will allow us to directly deduce the first 10 bits of $M_{9}$ that fulfill our extra constraint up to the $10^{\text {th }}$ bit (because knowing the first 24 bits of $M_{14}$ will lead to the first 24 bits of $X_{11}, X_{10}, X_{9}, X_{8}$ and the first 10 bits of $X_{7}$, which is exactly what we need according to equation (11)). Once a solution is found after $2^{3}$ tries on average, we can randomize the remaining $M_{14}$ unrestricted bits (the 8 most significant bits) and eventually deduce the 22 most significant bits of $M_{9}$ with equation (11). With this method, we completely remove the extra $2^{3}$ factor, because the cost is amortized by the final randomization of the 8 most significant bits of $M_{14}$.

Merging the branches with $M_{0}, M_{2}$ and $M_{5}$. Once $M_{9}$ and $M_{14}$ fixed, we still have message words $M_{0}, M_{2}$ and $M_{5}$ to determine for the merging. One can see that with only these three message words undetermined, all internal state values except $X_{2}, X_{1}, X_{0}, X_{-1}, X_{-2}, X_{-3}$ and $Y_{2}, Y_{1}, Y_{0}, Y_{-1}, Y_{-2}, Y_{-3}$ are fully known when computing backwards from the non-linear parts in each branch.

This is where our first constraint $Y_{3}=Y_{4}$ comes into play. Indeed, when writing $Y_{1}$ from the equation from step 4 in right branch, we have:

$$
Y_{1}=Y_{5}^{\ggg 13} \boxminus\left(Y_{4} \wedge Y_{2} \oplus Y_{3} \wedge \overline{Y_{2}}\right) \boxminus M_{9} \boxminus K_{0}^{r}=Y_{5}^{\ggg 13} \boxminus Y_{3} \boxminus M_{9} \boxminus K_{0}^{r}
$$

which means that $Y_{1}$ is already completely determined at this point (the bit condition present in $Y_{1}$ in Figure 4 is actually handled for free when fixing $M_{14}$ 
and $M_{9}$, since it requires to know the 9 first bits of $M_{9}$ ). In other words, the constraint $Y_{3}=Y_{4}$ allowed $Y_{1}$ to not depend on $Y_{2}$ which is currently undetermined. Another effect of this constraint can be seen when writing $Y_{2}$ from the equation from step 5 in right branch:

$Y_{2}=Y_{6}^{\ggg 15} \boxminus\left(Y_{5} \wedge Y_{3} \oplus Y_{4} \wedge \overline{Y_{3}}\right) \boxminus M_{2} \boxminus K_{0}^{r}=Y_{6}^{\ggg 15} \boxminus\left(Y_{5} \wedge Y_{3}\right) \boxminus M_{2} \boxminus K_{0}^{r}=C_{0} \boxminus M_{2}$

where $C_{0}=Y_{6}^{\ggg 15} \boxminus\left(Y_{5} \wedge Y_{3}\right) \boxminus K_{0}^{r}$ is a constant.

Our second constraint $X_{5}^{{ }^{5}} \boxminus M_{4}=0$ xffffffff is useful when writing $X_{1}$ and $X_{2}$ from the equations from step 4 and 5 in left branch

$$
\begin{aligned}
& X_{2}=X_{6}^{\ggg 8} \boxminus\left(X_{5} \oplus X_{4} \oplus X_{3}\right) \boxminus M_{5}=C_{1} \boxminus M_{5} \\
& X_{1}=X_{5}^{5_{5}} \boxminus\left(X_{4} \oplus X_{3} \oplus X_{2}\right) \boxminus M_{4}=\overline{X_{4}} \oplus X_{3} \oplus X_{2}=\overline{X_{4}} \oplus X_{3} \oplus\left(C_{1} \boxminus M_{5}\right)
\end{aligned}
$$

where $C_{1}=X_{6}^{\ggg 8} \boxminus\left(X_{5} \oplus X_{4} \oplus X_{3}\right)$ is a constant.

Finally, our ultimate goal for the merge is to ensure that $X_{-3}=Y_{-3}, X_{-2}=$ $Y_{-2}, X_{-1}=Y_{-1}$ and $X_{0}=Y_{0}$, knowing that all other internal states are determined when computing backwards from the non-linear parts in each branch, except $Y_{2}=C_{0} \boxminus M_{2}, X_{2}=C_{1} \boxminus M_{5}$ and $X_{1}=\overline{X_{4}} \oplus X_{3} \oplus\left(C_{1} \boxminus M_{5}\right)$. We therefore write the equations relating these eight internal state words:

$$
\begin{aligned}
& X_{0}=X_{4}^{\gg 12} \boxminus\left(X_{3} \oplus X_{2} \oplus X_{1}\right) \boxminus M_{3}=X_{4}^{\gg 12} \boxminus \overline{X_{4}} \boxminus M_{3} \\
& =Y_{0}=Y_{4}^{\ggg 11} \boxminus\left(Y_{3} \wedge Y_{1} \oplus Y_{2} \wedge \overline{Y_{1}}\right) \boxminus M_{0} \boxminus K_{0}^{r}=Y_{4}^{\gg 11} \boxminus\left(Y_{3} \wedge Y_{1} \oplus\left(C_{0} \boxminus M_{2}\right) \wedge \overline{Y_{1}}\right) \boxminus M_{0} \boxminus K_{0}^{r} \\
& X_{-1}=X_{3}^{\ggg 15} \boxminus\left(X_{2} \oplus X_{1} \oplus X_{0}\right) \boxminus M_{2}=X_{3}^{\ggg 15} \boxminus\left(\overline{X_{4}} \oplus X_{3} \oplus X_{0}\right) \boxminus M_{2} \\
& =Y_{-1}=Y_{3}^{\ggg 9} \boxminus\left(Y_{2} \wedge Y_{0} \oplus Y_{1} \wedge \overline{Y_{0}}\right) \boxminus M_{7} \boxminus K_{0}^{r}=Y_{3}^{\ggg 9} \boxminus\left(\left(C_{0} \boxminus M_{2}\right) \wedge X_{0} \oplus Y_{1} \wedge \overline{X_{0}}\right) \boxminus M_{7} \boxminus K_{0}^{r} \\
& X_{-2}=X_{2} \ggg 14 \boxminus\left(X_{1} \oplus X_{0} \oplus X_{-1}\right) \boxminus M_{1}=\left(C_{1} \boxminus M_{5}\right) \ggg 14 \boxminus\left(\overline{X_{4}} \oplus X_{3} \oplus\left(C_{1} \boxminus M_{5}\right) \oplus X_{0} \oplus X_{-1}\right) \boxminus M_{1} \\
& =Y_{-2}=Y_{2}^{\ggg 9} \boxminus\left(Y_{1} \wedge Y_{-1} \oplus Y_{0} \wedge \overline{Y_{-1}}\right) \boxminus M_{14} \boxminus K_{0}^{r}=\left(C_{0} \boxminus M_{2}\right) \ggg 9 \boxminus\left(Y_{1} \wedge X_{-1} \oplus X_{0} \wedge \overline{X_{-1}}\right) \boxminus M_{14} \boxminus K_{0}^{r} \\
& X_{-3}=X_{1} \ggg 11 \boxminus\left(X_{0} \oplus X_{-1} \oplus X_{-2}\right) \boxminus M_{0}=\left(\overline{X_{4}} \oplus X_{3} \oplus\left(C_{1} \boxminus M_{5}\right)\right) \ggg 11 \boxminus\left(X_{0} \oplus X_{-1} \oplus X_{-2}\right) \boxminus M_{0} \\
& =Y_{-3}=Y_{1}^{\gg 8} \boxminus\left(Y_{0} \wedge Y_{-2} \oplus Y_{-1} \wedge \overline{Y_{-2}}\right) \boxminus M_{5} \boxminus K_{0}^{r}=Y_{1}^{\ggg 8} \boxminus\left(X_{0} \wedge X_{-2} \oplus X_{-1} \wedge \overline{X_{-2}}\right) \boxminus M_{5} \boxminus K_{0}^{r}
\end{aligned}
$$

If these four equations are verified, then we have merged left and right branch to the same input chaining variable. We first remark that $X_{0}$ is already fully determined and thus the second equation $X_{-1}=Y_{-1}$ only depends on $M_{2}$. Moreover, it is a T-function in $M_{2}$ (any bit $i$ of the equation depends only on the $i$ first bits of $M_{2}$ ) and can therefore be solved very efficiently bit per bit. We explain in the full version how to solve this T-function and our average cost in order to find one $M_{2}$ solution is one RIPEMD-128 step computations.

Since $X_{0}$ is already fully determined, from the $M_{2}$ solution previously obtained we directly deduce the value of $M_{0}$ to satisfy the first equation $X_{0}=Y_{0}$. From $M_{2}$ we can compute the value of $Y_{-2}$ and we know that $X_{-2}=Y_{-2}$ and we calculate $X_{-3}$ from $M_{0}$ and $X_{-2}$. At this point, the two first equations are fulfilled and we still have the value of $M_{5}$ to choose.

The third equation can be rewritten $V^{\ggg 14}=\left(V \oplus C_{2}\right) \boxplus C_{3}$, where $V=X[2]=$ $\left(C_{1} \boxminus M_{5}\right)$ and $C_{2}, C_{3}$ are two constants. Similarly, the fourth equation can be rewritten $V^{\ggg 11}=\left(V \boxplus C_{4}\right) \oplus C_{5}$, where $C_{4}, C_{5}$ are two constants. Solving either 
of these two equations with regards to $V$ can be costly because of the rotations, so we combine them to create simpler one: $\left(\left(V \oplus C_{2}\right) \boxplus C_{3}\right) \lll 3=\left(V \boxplus C_{4}\right) \oplus C_{5}$. This equation is easier to handle because the rotation coefficient is small: we guess the 3 most significant bits of $\left(\left(V \oplus C_{2}\right) \boxplus C_{3}\right)$ and we solve simply the equation 3-bit layer per 3-bit layer, starting from the least significant bit. From the value of $V$ deduced, we straightforwardly obtain $M_{5}=C_{1} \boxminus V$ and the cost of recovering $M_{5}$ is equivalent to 8 RIPEMD-128 step computations (the 3-bit guess implies a factor of 8 , but the resolution can be implemented very efficiently with tables).

When all three message words $M_{0}, M_{2}$ and $M_{5}$ have been fixed, the first, second and a combination of the third and fourth equalities are necessarily verified. However, we have a probability $2^{-32}$ that both the third and fourth equations will be fulfilled. Moreover, one can check in Figure 4 that there is one bit condition on $X_{0}=Y_{0}$ and one bit condition on $Y_{2}$ and this further adds up a factor $2^{-2}$. We evaluate the whole process to cost about 19 RIPEMD-128 step computations on average: there are 17 steps to compute backwards after having identified a proper couple $M_{14}, M_{9}$, and the 8 RIPEMD-128 step computations to obtain $M_{5}$ are only done $1 / 4$ of the time because the two bit conditions on $Y_{2}$ and $X_{0}=Y_{0}$ are filtered before.

To summarize the merging: we first compute a couple $M_{14}, M_{9}$ that satisfies a special constraint, we find a value of $M_{2}$ that verifies $X_{-1}=Y_{-1}$, then we directly deduce $M_{0}$ to fulfill $X_{0}=Y_{0}$, and we finally obtain $M_{5}$ to satisfy a combination of $X_{-2}=Y_{-2}$ and $X_{-3}=Y_{-3}$. Overall, with only 19 RIPEMD-128 step computations on average, one can merge the branches with probability $2^{-34}$.

\section{$5 \quad$ Results and Implementation}

\subsection{Complexity Analysis and Implementation}

After the quite technical description of the attack in previous section, we would like to rewrap everything to get a clearer view of the attack complexity, the amount of freedom degrees etc. Given a starting point from phase 2, the attacker can perform $2^{26}$ merge processes (because 3 bits are already fixed in both $M_{9}$ and $M_{14}$, and the extra constraint consumes 32 bits) and since one merge process succeeds only with probability of $2^{-34}$, he obtains a solution with probability $2^{-8}$. Since he needs $2^{30.32}$ solutions from the merge to have a good chance to verify the probabilistic part of the differential path, a total of $2^{38.32}$ starting points will have to be generated and handled.

From the end of phase 1 , he generates $2^{38.32}$ starting points in phase 2 , that is $2^{38.32}$ differential paths like the one from Figure 4 (with the same step probabilities). For each of them, in phase 3 he tries $2^{26}$ times to find a solution for the merge with an average complexity of 19 RIPEMD-128 step computations for each try. The SFS collision final complexity is $19 \cdot 2^{26+38.32}$ RIPEMD-128 step computations, which corresponds to $(19 / 128) \cdot 2^{64.32}=2^{61.57}$ RIPEMD-128 compression function computations (there are 64 steps in each branch). 
The merge process has been implemented and we give in the full version of the article an example of a message and chaining variable couple that verifies the merge (i.e. they follow the differential path from Figure 3 until step 25 of the left branch and step 20 of the right branch). We measured the efficiency of our implementation in order to confront it to our theoretic complexity estimation. As point of reference, we observed that on the same computer, an optimized implementation of RIPEMD-160 (OpenSSL v.1.0.1c) performs $2^{21.44}$ compression function computations per second. With 4 rounds instead of 5 and about $3 / 4$ less operations per step, we extrapolated that RIPEMD-128 would perform at $2^{22.17}$ compression function computations per second. Our implementation performs $2^{24.61}$ merge process (both phase 2 and phase 3 ) per second on average, which therefore corresponds to a SFS collision final complexity of $2^{61.88}$ RIPEMD-128 compression computations. While our practical results confirm our theoretical estimations, we emphasize that the latter are a bit pessimistic, since our attack implementation is not optimized. As a side note, we also verified experimentally that the probabilistic part in both left and right branch can be fulfilled.

A last point needs to be checked: the complexity estimation for the generation of the starting points. Indeed, as much as $2^{38.32}$ starting points are required at the end of phase 2 and the algorithm being quite heuristic, it is hard to analyze precisely. The amount of freedom degrees is not an issue since we already saw in Section 4.1 that about $2^{306.91}$ solutions are expected to exist for the differential path at the end of phase 1 . A completely new starting point takes about 5 minutes to be outputted on average with our implementation, but from one such path we can directly generate $2^{18}$ equivalent ones by randomizing $M_{7}$. Using the OpenSSL implementation as reference, this amounts to $2^{50.72}$ RIPEMD-128 computations to generate all the starting points that we need in order to find a SFS collision. This gross estimation is extremely pessimistic since its doesn't even take in account the fact that once a starting point is found, one can also randomize $M_{4}$ and $M_{11}$ to find many other valid candidates with a few operations. Finally, one may argue that with this method the starting points generated are not independent enough (in backward direction when merging and/or in forward direction for verifying probabilistically the linear part of the differential path). However, no such correlation was detected during our experiments and previous attacks on similar hash functions 1314 showed that only a few rounds were enough to observe independence between bit conditions. In addition, even if some correlations existed, since we are looking for many solutions, the effect would be averaged among good and bad candidates.

Collision for the RIPEMD-128 Compression Function. We described in previous sections a SFS collision attack for the full RIPEMD-128 compression function with $2^{61.57}$ computations. It is clear from Figure 4 that we can remove the 4 last steps of our differential path in order to attack a 60-step reduced variant of the RIPEMD-128 compression function. No difference will be present in the internal state at the end of the computation and we directly get a collision, saving a factor $2^{4}$ over the full RIPEMD-128 attack complexity. 
We also give in the full version of the article a slightly different freedom degrees utilization when attacking 63 steps of the RIPEMD-128 compression function (the first step being taken out), that saves a factor $2^{1.66}$ over the collision attack.

Distinguishers. We provide in the full version of this article a limited-birthday distinguisher [11] on the full RIPEMD-128 compression but also hash function.

\section{Conclusion}

In this article, we proposed a new cryptanalysis technique for RIPEMD-128, that led to a collision attack on the full compression function as well as a distinguisher for the full hash function. We believe that our method still presents room for improvements, and we expect a practical collision attack for the full RIPEMD-128 compression function to be found during the incoming years. While our results don't endanger the collision resistance of the RIPEMD-128 hash function as a whole, we emphasize that SFS collision attacks are a strong warning sign which indicates that RIPEMD-128 might not be as secure as the community expected. Considering the history of the attacks on the MD5 compression function [78], MD5 hash function [29], and then MD5-protected certificates [24, we believe that another function than RIPEMD-128 should be used for new security applications. Future works include reducing the attack complexity and applying our methods to RIPEMD-160 and other parallel branches-based functions.

\section{References}

1. Bertoni, G., Daemen, J., Peeters, M., Van Assche, G.: Keccak specifications. Submission to NIST (2008)

2. Bosselaers, A., Preneel, B. (eds.): RIPE 1992. LNCS, vol. 1007. Springer, Heidelberg (1995)

3. Brassard, G. (ed.): CRYPTO 1989. LNCS, vol. 435. Springer, Heidelberg (1990)

4. De Cannière, C., Rechberger, C.: Finding SHA-1 Characteristics: General Results and Applications. In: Lai, X., Chen, K. (eds.) ASIACRYPT 2006. LNCS, vol. 4284, pp. 1-20. Springer, Heidelberg (2006)

5. Cramer, R. (ed.): EUROCRYPT 2005. LNCS, vol. 3494. Springer, Heidelberg (2005)

6. Damgård, I.: A Design Principle for Hash Functions. In: Brassard, pp. 416-427

7. den Boer, B., Bosselaers, A.: Collisions for the Compression Function of MD5. In: Helleseth, T. (ed.) EUROCRYPT 1993. LNCS, vol. 765, pp. 293-304. Springer, Heidelberg (1994)

8. Dobbertin, H.: Cryptanalysis of MD5 compress. In: Rump Session of Advances in Cryptology EUROCRYPT 1996 (1996)

9. Dobbertin, H.: RIPEMD with Two-Round Compress Function is Not CollisionFree. J. Cryptology 10(1), 51-70 (1997)

10. Dobbertin, H., Bosselaers, A., Preneel, B.: RIPEMD-160: A Strengthened Version of RIPEMD. In: Gollmann, D. (ed.) FSE 1996. LNCS, vol. 1039, pp. 71-82. Springer, Heidelberg (1996) 
11. Gilbert, H., Peyrin, T.: Super-Sbox Cryptanalysis: Improved Attacks for AES-Like Permutations. In: Hong, S., Iwata, T. (eds.) FSE 2010. LNCS, vol. 6147, pp. 365383. Springer, Heidelberg (2010)

12. ISO. ISO/IEC 10118-3:2004: Information technology — Security techniques Hash-functions - Part 3: Dedicated hash-functions. pub-ISO (February 2004)

13. Joux, A., Peyrin, T.: Hash Functions and the (Amplified) Boomerang Attack. In: Menezes, A. (ed.) CRYPTO 2007. LNCS, vol. 4622, pp. 244-263. Springer, Heidelberg (2007)

14. Manuel, S., Peyrin, T.: Collisions on SHA-0 in One Hour. In: Nyberg, K. (ed.) FSE 2008. LNCS, vol. 5086, pp. 16-35. Springer, Heidelberg (2008)

15. Mendel, F., Nad, T., Scherz, S., Schläffer, M.: Differential Attacks on Reduced RIPEMD-160. In: Gollmann, D., Freiling, F.C. (eds.) ISC 2012. LNCS, vol. 7483, pp. 23-38. Springer, Heidelberg (2012)

16. Mendel, F., Nad, T., Schläffer, M.: Collision Attacks on the Reduced Dual-Stream Hash Function RIPEMD-128. In: Canteaut, A. (ed.) FSE 2012. LNCS, vol. 7549, pp. 226-243. Springer, Heidelberg (2012)

17. Mendel, F., Pramstaller, N., Rechberger, C., Rijmen, V.: On the Collision Resistance of RIPEMD-160. In: Katsikas, S.K., López, J., Backes, M., Gritzalis, S., Preneel, B. (eds.) ISC 2006. LNCS, vol. 4176, pp. 101-116. Springer, Heidelberg (2006)

18. Merkle, R.C.: One Way Hash Functions and DES. In: Brassard [3], pp. 428-446

19. Ohtahara, C., Sasaki, Y., Shimoyama, T.: Preimage Attacks on Step-Reduced RIPEMD-128 and RIPEMD-160. In: Lai, X., Yung, M., Lin, D. (eds.) Inscrypt 2010. LNCS, vol. 6584, pp. 169-186. Springer, Heidelberg (2011)

20. Rivest, R.L.: The MD4 message-digest algorithm. Request for Comments (RFC) 1320, Internet Activities Board, Internet Privacy Task Force (April 1992)

21. Sasaki, Y., Aoki, K.: Meet-in-the-Middle Preimage Attacks on Double-Branch Hash Functions: Application to RIPEMD and Others. In: Boyd, C., González Nieto, J. (eds.) ACISP 2009. LNCS, vol. 5594, pp. 214-231. Springer, Heidelberg (2009)

22. Sasaki, Y., Wang, L.: Distinguishers beyond Three Rounds of the RIPEMD-128/160 Compression Functions. In: Bao, F., Samarati, P., Zhou, J. (eds.) ACNS 2012. LNCS, vol. 7341, pp. 275-292. Springer, Heidelberg (2012)

23. Shoup, V. (ed.): CRYPTO 2005. LNCS, vol. 3621. Springer, Heidelberg (2005)

24. Stevens, M., Sotirov, A., Appelbaum, J., Lenstra, A., Molnar, D., Osvik, D.A., de Weger, B.: Short Chosen-Prefix Collisions for MD5 and the Creation of a Rogue CA Certificate. In: Halevi, S. (ed.) CRYPTO 2009. LNCS, vol. 5677, pp. 55-69. Springer, Heidelberg (2009)

25. Wang, X., Yu, H., Lisa Yin, Y.: Efficient Collision Search Attacks on SHA-0. In: Shoup [23], pp. 1-16

26. Wang, L., Sasaki, Y., Komatsubara, W., Ohta, K., Sakiyama, K. (Second) Preimage Attacks on Step-Reduced RIPEMD/RIPEMD-128 with a New Local-Collision Approach. In: Kiayias, A. (ed.) CT-RSA 2011. LNCS, vol. 6558, pp. 197-212. Springer, Heidelberg (2011)

27. Wang, X., Lai, X., Feng, D., Chen, H., Yu, X.: Cryptanalysis of the Hash Functions MD4 and RIPEMD. In: Cramer [5], pp. 1-18

28. Wang, X., Lisa Yin, Y., Yu, H.: Finding Collisions in the Full SHA-1. In: Shoup [23], pp. 17-36

29. Wang, X., Yu, H.: How to Break MD5 and Other Hash Functions. In: Cramer [5], pp. $19-35$ 